\title{
Density maps of geophysical study as a new type of integrated characteristic of geophysical fields in atlases
}

\author{
Loginov D.S. ${ }^{a, b}$, Krylov S.A. ${ }^{b}$ \\ ${ }^{a}$ Pangea Inc., Moscow, Russian Federation; loginov_ds@pangea.ru,loginov@cartlab.ru \\ ${ }^{b}$ Moscow State University of Geodesy and Cartography (MIIGAiK), Moscow, Russian Federation; krylov@cartlab.ru \\ * Corresponding author
}

\begin{abstract}
Nowadays the geophysical section of traditional atlases is not sufficiently informative. The amount of geophysical fields' maps rarely exceeds two. On the other hand, the electronic GIS atlases invariably include in their composition maps of study of geophysical observations, which make it possible to assess the level of exploration on the mapped territory. The creation and design of such maps today are limited to displaying only the factual location of observation profiles and other objects. In order to expand the possibilities of analyzing geophysical information, the authors suggests a method for creating of density maps of geophysical study. The study on these maps is shown with isolines - usual method for geophysical maps. The authors of this article describe the method of creating density maps of geophysical study. This method includes the following steps: selection and analysis of information sources of geophysical study; choice of study parameters for mapping; calculation of the density of objects of geophysical study; interpolation and creation of isolines of objects of geophysical study density. The created study maps of density dynamics represent the approbation of the method; they allow increasing the information content of geophysical section of the atlas. The study is also a special factor that determines general need of mapping the phenomenon and characterizes the availability of data sources for mapping. Therefore, the opportunities for using developed maps for the automated determination of the atlas structure are also shown in the article.
\end{abstract}

Keywords: geophysical study, density maps, atlas mapping, geophysical maps, GIS-atlases

\section{Geophysical maps in modern atlases}

At the present time, the atlas mapping is rapidly progressing towards the development of geo-information and geo-portal solutions. It is especially true for complex atlases, where systematic information about natural and socio-economic phenomena of the mapped territory is provided.

Due to the growth of data published in open access, as well as due to active use of modern technologies the problem of increasing atlas information content arises. It can clearly be seen in the geophysical section of an atlas. As a rule, there are a limited number of maps in this section that do not allow providing a comprehensive characteristic of geophysical fields within the mapped territory. Atlases with more than two geophysical maps are exceptions rather than a rule. National Atlases of the Czech Republic, Spain and Japan can be attributed to most complete atlases by the level of informational content of geophysical section. They contain maps of several types of geophysical fields. For example, the Natural Landscape section of National Atlas of the Czech Republic includes maps of the geomagnetic field, the radiometric field, the gravitation field and the temperature at a depth under surface. Maps of gravity, magnetic, seismic fields, as well as some maps of geophysical study are presented in the National Atlas of Spain. It is also worth noting the examples where the composition of the geophysical section is expanded due to the large number of maps of several fields. For example, there are six geophysical maps in the Physical features section of the National Atlas of Japan (in both editions of 1977 and of 1990): maps of two types of gravity field anomalies (Bouguer and Free Air), as well as the Total Intensity map of the magnetic field and its three components (Horizontal, Declination, Inclination). In addition to traditional atlases the analysis of geological and geophysical GIS atlases (Loginov, 2016) was conducted. According to its results, it was determined that the main type of geophysical maps today are study maps for various methods of geophysical observations the study is presented in 10 of 12 analysed atlases. This trend suggests that study maps should also be included in the sections of traditional atlases. However, the implementation of such a decision should be based on analysis of the level of study of the territory, since the more information that is available on the phenomenon being mapped the more important it is to include the map in the atlas. Therefore, for this implementation it is necessary to learn the experience of study mapping and evaluate the possibility of using existing maps to assess study as one of the factors for inclusion of maps in the atlas.

\subsection{Current methods of geophysical study mapping}

Geophysical study denotes the assessment of the presence and distribution of geological and geophysical objects 
and infrastructure facilities of exploration surveys, as well as an assessment of coverage of the territory by survey networks, cartographic materials and so forth. Geophysical study assessment is an important step in justifying the formulation of geological exploration, therefore compilations of study maps is a mandatory element of any geophysical work. As a rule, the information about study is presented in two forms:

a) Maps of geophysical study (Figure 1) display the actual state of the exploration infrastructure: local and regional survey profiles, observation points, boreholes, as well as subsoil use areas, etc.

b) Generalized study maps and schemes of areal data (Figure 2) provide information on the extent and quality of coverage of performed field works, and on cartographic support of geophysical surveys by drawing the spatial boundaries of the map sheets contours or boundaries of aerial surveys on the general geographical basis.

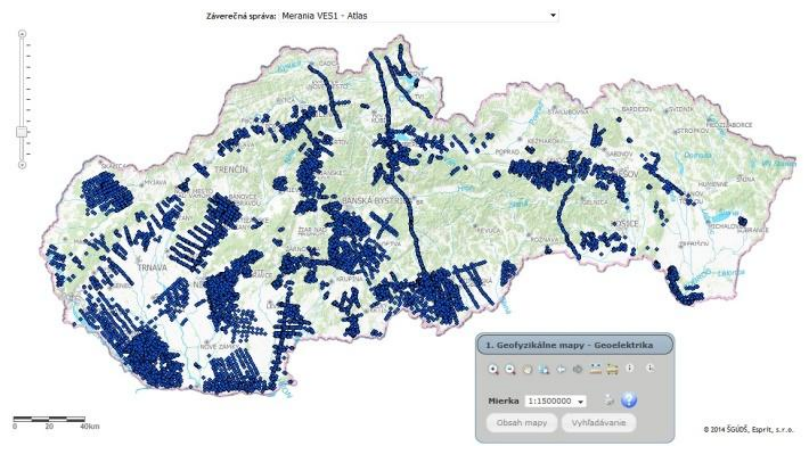

Figure 1. Map of Vertical electrical sounding (VES) measurements in Slovakia by SGIDS (C) Map Portal (SGIDŠ) 2014.

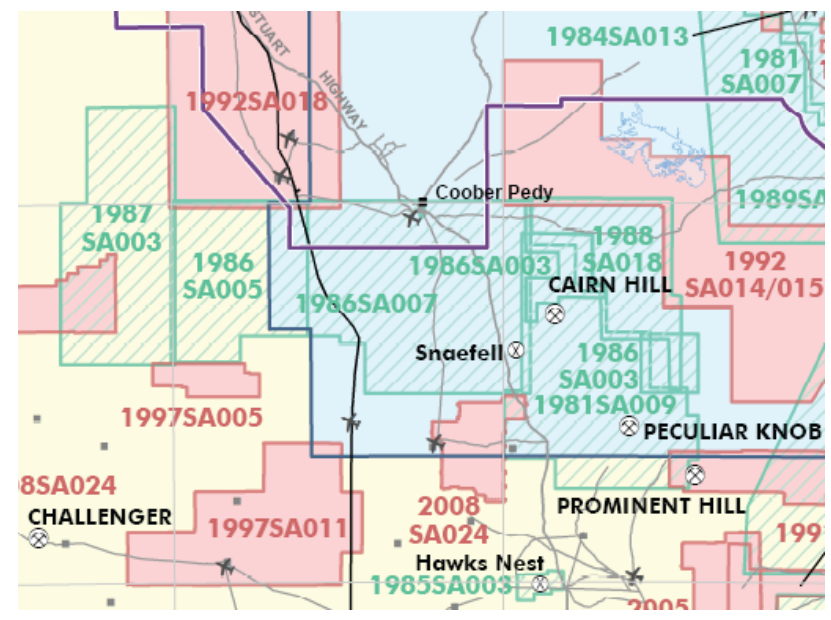

Figure 2 - Fragment of «Company airborne geophysical surveys» map: years of Airborne MAG/RAD company survey is shown by different color of areas (C) Government of South Australia 2018

The information of geophysical study also can be referred as one method of geophysical surveys (e.g. maps of the study by airborne magnetic survey) or several methods (e.g. maps of gravity and magnetic survey study). Contour or filling color corresponds to one of parameters of survey such as type of survey, time, scale, accuracy etc. The combination of several parameters on the map causes the use of a large number of graphical variables to ensure a separate perception of objects and makes it difficult to make a visual assessment of phenomenon.

Thus, current methods of geophysical study displaying are reduced to the actual display of objects location. However, this form of representation may not be enough in relation to integrated characteristics of mapped territory and also for solving problems of automated decision making on the inclusion of maps in the atlas. To automate the decision to include a single map into the atlas with taking into account the level of phenomenon study, the most optimal way of showing the study is the isoline method.

\subsection{Display of geophysical study in isoline maps form}

Geophysical study can be represented in the form of field maps, if we turn to the concept of the density of study objects. This assumption does not contradict the subject of mapping, since study can be assessed as an abstract concept arising at the stage of generalization of information. In this case, it is possible to apply special methods of cartographic-morphometric (isolinear) modelling for mapping of the density of geophysical study.

Mapping of density fields of discrete indicators is actively used in geographical research. However, in geophysics there are a few examples of analytical maps of density where only density of observation profiles is shown by pseudo-isolines. Such maps allow the evaluation the general level of study in a visual and «familiar» isolinear form during the initial acquaintance with the mapped territory. Depending on the study parameter, isolines can transmit the density of surveys of various scale, accuracy, time, etc., which significantly increases the types of geophysical maps. Such maps can serve as an excellent complement to the geophysical section of atlases.

To analyze the cartographic image of geophysical fields, the authors propose to display the density of objects of study with various localization patterns (point, linear, areal) on maps with using of contour lines. This method of cartographic images allows to simultaneously maintain the continuity of the image field and increase the information content of the map.

\section{Method of geophysical study density maps creation}

In accordance with the proposed approach of displaying geophysical study using the isolines method, the following method is proposed for creating density maps of geophysical study:

a) Selection and analysis of information sources of geophysical study;

b) Choice of study parameters for mapping;

c) Calculation of the density of objects of geophysical study; 
d) Interpolation and creation of isolines of objects of geophysical study density.

Each stage will be reviewed in more detail further.

\subsection{Selection and analysis of information sources of geophysical study}

The sets of geophysical surveys data conducted by various methods of exploratory geophysics are the source of information for creating isolines of the density of geophysical study. Currently, this information can be obtained with specialized geo-portals and GIS atlases.

Geophysical study data are represented by vector spatial objects of three types of localization: point (e.g. observation points, wells), linear (observation profiles) and area (contours of aerial surveys, etc.). Of course, there is only part of information that can be, for one reason or another, laid out in open access. Therefore, it would be good to use complete data for the most objective assessment of study. Nevertheless, the information that is provided in the open form may be enough to get a general representation about the study in the mapped area, which is the purpose of maps placed in atlases.

\subsection{Choice of study parameters for mapping}

The vector representation of information allows the use of data in several studies, especially if the attribute information is complete. For geophysical study, the required fields of table are the «report name», «work method», «time survey», «survey scale», «implementing organization», etc. Depending on the purpose of future map, the key attribute is selected.

With regard to the development of the density map of geophysical study in the atlas, it is advisable to use the «survey scale» parameter, which is directly related to the detail of surveys and, accordingly, the level and quality of territory studied. The larger the scales of survey, the more observation points were within the contour of work, and the more accurately the characteristics of geophysical field were observed. The «work method» parameter differentiates information according to the method of exploratory geophysics: gravity survey, magnetic survey, seismic survey, etc. The additional «time survey» parameter makes it possible to create a series of maps that show the dynamics of the study level for specific time periods.

\subsection{Calculation of the density of objects of geophysical study}

The density of objects of study can be calculated in several ways. The first, and most simple, is to quickly assess the density of data layer objects using GIS tools. For example, ArcToolbox has at least three density tools: Kernel Density, Line Density and Point Density. However, this rapid assessment is associated with the need to choose the most appropriate smoothing parameter, because default setting does not take into account the specifics of mapping phenomenon. In addition, not all tools works with area objects and most of them are among geophysical study.
In this regard, it is proposed to calculate the density of objects of geophysical study by their density per unit area using a hexagonal pallet. The choice of this type of palette is based on the fact that the isoline density maps have the ability to transfer the mean values of density for an arbitrary area and its gradient, if the averaging cell used in the compilation has the shape of a centrally symmetric quadrangle or hexagon in the making process. Since the field observations takes place in space-limited sites, and the maps made are of rather small scale, it is advisable to cover the mapped area with a uniform network of hexagons. To determine the optimal width of hexagonal grid cells, depending on the scale of result map, it is possible to use the following relation, calculated empirically:

$$
W=0,3 M
$$

where $\mathrm{W}=$ hexagonal grid width, in $\mathrm{km}$ $\mathrm{M}=$ scale denominator expressed in $\mathrm{km}$ per

$1 \mathrm{~cm}$.

After that, the geometric centers of the cells are calculated and the density value calculated inside the grid cell is written to it. A uniform point network derived from cell centroids serves as the basis for creation of a digital model of parameter.

\subsection{Interpolation and creation of isolines of objects of geophysical study density}

Point data with information about density of study objects allow the use of interpolation methods to recreate the field of study. The most optimal method is kriging, which allows reproducing the values at the existing points with minimal variation in interpolation error. However, the use of Interpolation with barriers (i.e. Kernel Smoothing) methods implemented in Geostatistical Analyst (ArcGIS) also provides optimal results. The result of interpolation is a matrix network and density isolines of objects of study. Isolines can be smoothed for a more expressive display on the map.

It is worth noting that when assessing study, information of all types of localization is taken into account. For convenience of further density calculations, it is necessary to assign a certain amount of point data to linear and area objects in order to bring the available information into a single format convenient for the subsequent steps of the method. It can be done by knowing the national regulatory requirements for the density of geophysical observation points, depending on the scale and method of survey.

\subsection{Testing of method}

In the present study, two subjects of the Russian Federation, located in different geological and geophysical conditions were chosen as the mapping territory: the Khanty-Mansi Autonomous District (KhMAO) and the Voronezh Region. Open data of geophysical study on the area nature of localization (the contours of airborne and surface gravimetric observations) were used as the initial data. For this data, the scale and survey year are known, so the final maps 
will reflect the density dynamics of gravimetric observations. The scales of the result maps are 1:15000 000 and 1:5000 000 respectively - therefore the width of the hexagonal grid cell according to the formula (1) is 45 and $15 \mathrm{~km}$.

To build a network of regular hexagons, an additional module Repeating Shapes by Jenness Enterprises (Jenness, J. 2012) was used for ArcGIS. It is allows to specify hexagons' width, orientation and degrees offset both on the set of objects in the layers and on the selected objects. As a method of creation, a polygon with a subject's boundary was pre-allocated, after which the desired parameters of the network of hexagons were entered.

Next, the number of polygons of gravimetric surveys with different scales is calculated for each grid cell. In conditions of a limited amount of information, it is fair to accept the following condition: within a polygonal object of study, where the survey was carried out with a certain scale, such a number of observation points per 1 sq. $\mathrm{km}$ is evenly distributed, which is provided for by the Gravity Survey Instructions for the corresponding survey scale. Thus, if a hexagonal grid cell intersects one polygon or profile of 1:50 000 scale gravimetric survey, then the centroid of the cell is assigned a network density equal to 30 points per 1 sq.km (Figure 3 ).
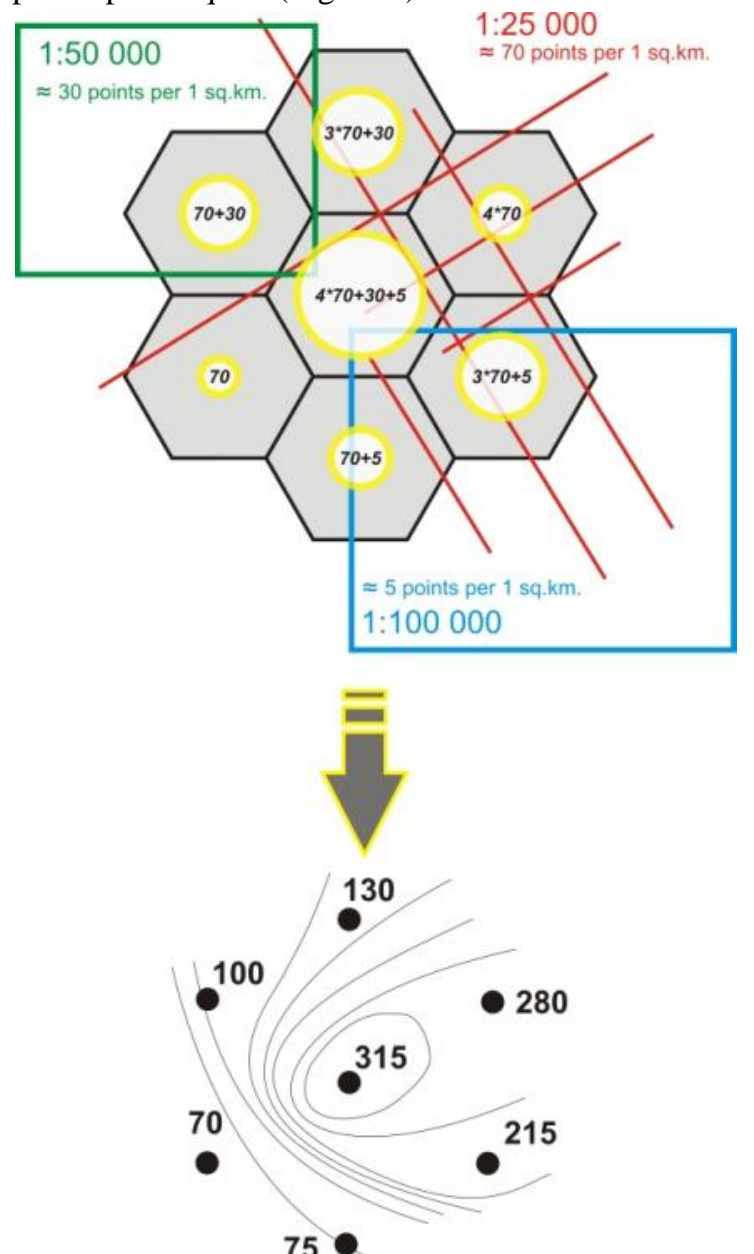

Figure 3 - Process of density of geophysical study calculation (C) Loginov D.S., Krylov S.A. 2018
As a result, a database is formed, which contains information about the study density in different time periods. This information serves as the basis for creation the digital field model in the manner of Kernel Smoothing. Design of result grids is made in ArcGIS. The data within the selected time range was used in the construction of each map. The isolines of study density make it possible to estimate the quantity and quality of surveys, as well as the overall level of study of the territory.

Figures 4 and 5 present examples of the author's series of dynamics of gravimetric observations.

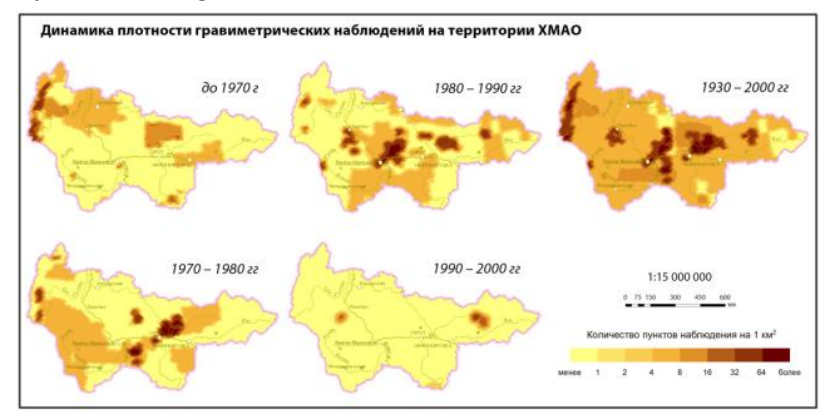

Figure 4 - Map series «Dynamics of density of gravimetric observations on Khanty-Mansi Autonomous District» (C) Loginov D.S., Krylov S.A. 2018

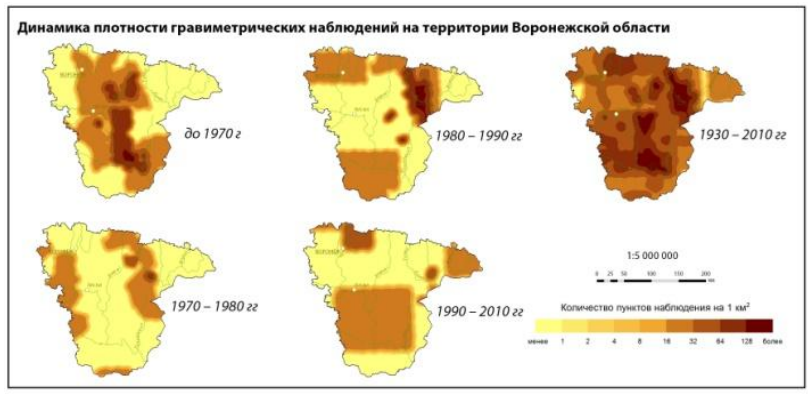

Figure 5 - Map series «Dynamics of density of gravimetric observations on Voronezh Region» (C) Loginov D.S., Krylov S.A. 2018

Analysis of the map series allows to determine the history of gravimetric work in the territory of the Russian Federation regions. According to the map, in the greater part of the territory of KhMAO and Voronezh region, there are observed higher density of observations. Such series of maps can be included in the atlas as independent map series, which allows significantly expanding the composition and increasing the information content of the geophysical section of atlases.

\section{Use of density maps of geophysical study in the development of atlas structure}

The proposed maps of the density of geophysical study determine the overall feasibility of mapping the field and characterize the availability of data sources for mapping. Thus, the more sources of information about a phenomenon that are available, the more likely is the need for its mapping in order to provide a comprehensive assessment of the territory. At the same time, it is possible to note the high level of automation of the process of creating such maps, which allows them to be 
obtained online. To do this, it is possible to use the database of main and additional sources of information, where the source data, the source for obtaining them, the update date, key indicators, etc. are defined.

Above mentioned opportunities allow the use of the developed density maps of geophysical study as a means for the automated development of the structure of atlas geophysical section. So on the basis of the density map data, one can estimate the percentage of territory covered by a large number of surveys. To do this, the map is converted to polygonal view, where the polygons will be the intervals between the contour lines. Then each scale range is assigned a point: low study -1 point, high -5 points. If there are areas where points are from 3 to 5 , then the map is included in the atlas.

Below are examples of converting density maps to point (scores) maps. From figure 6 it can be seen that the low indicator of the «study» criterion gives a recommendation for not including the map of gravity field in the atlas of the Khanty-Mansi Autonomous District. On the contrary, for the Voronezh region, the points above 3 exceed more than half of the territory's area, which means that the map should be included in the atlas.

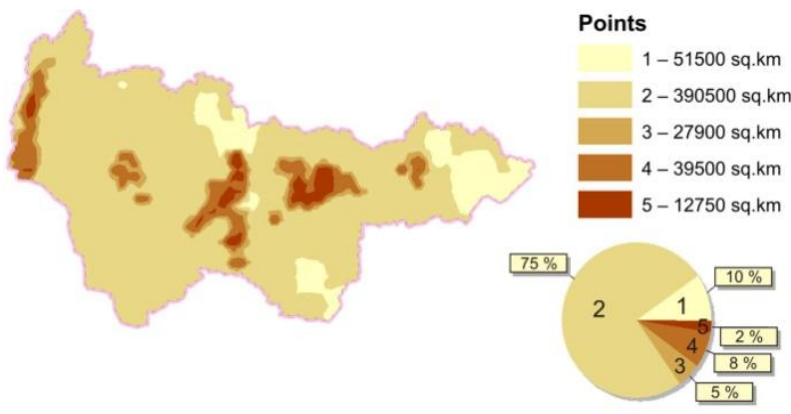

Figure 6 - Points (scores) map of gravity study on KhantyMansi Autonomous District (C) Loginov D.S., Krylov S.A. 2018

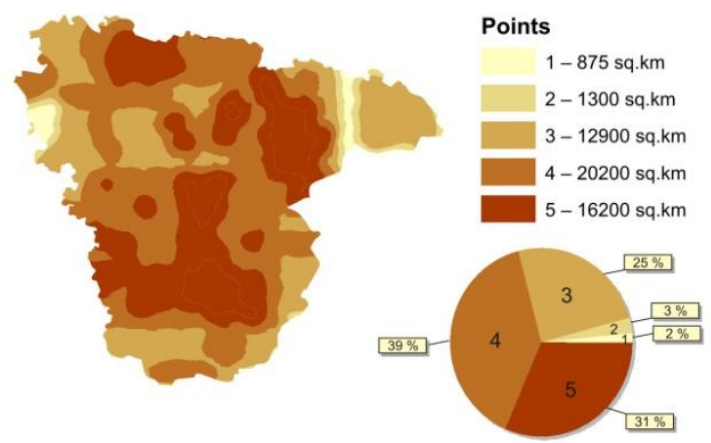

Figure 7 - Points (scores) map of gravity study on Voronezh Region (C) Loginov D.S., Krylov S.A. 2018

However, it should be noted that only the data on the geophysical study of the field are not enough for the final decision on inclusion of corresponding map in the atlas. In accordance with the concept of automated creation of atlases developed at the Moscow State University of Geodesy and Cartography, it is suggested that other factors be taken into account when developing the structure of atlas (Krylov et al., 2018). For example, the decision to include a particular geophysical map in the atlas is most influenced by the following factors: diversity level of geophysical field anomalies; character of relief; mineral resources distribution; position of territory in relation to seismic activity areas; others. Therefore, despite the fact that the Voronezh Region is well studied, the mineral resources distribution significantly reduces the chances of including maps in the atlas, since the region does not have large hydrocarbon reserves. On the contrary, the Khanty-Mansi Autonomous District is the largest region of Russia for the extraction of hydrocarbons; therefore the factor «mineral resources distribution» will correct the possibility of including the map of gravity field in the atlas. In addition, such a low density of gravimetric observations could be due to the insufficient completeness of the original open data.

\section{Results}

According to the results of research, the following conclusions can be drawn:

- The new approach proposed to modelling geophysical study through the conversion from the display of actual location of surveys to the isolinear representation of the density of geophysical observations objects;

- The method for mapping the density of geophysical study was developed and tested. Depending on the indicator used, it is possible to create a series of maps, the analysis of which allows estimating the history of geophysical operations in the mapped area. The proposed maps should be included in geophysical section of the atlases as independent maps for integrated characteristic of geophysical fields;

- The possibility of taking into account the level of study and use of the proposed density maps for the automated development of atlas structure is considered in the article. In the future, it is planned to jointly take maps of the density of geophysical study with maps of other factors for the final decision on composition of geophysical section of atlas.

\subsection{Acknowledgements}

The study was financially supported by the Ministry of Education and Science of the Russian Federation, grant No. 5.8029.2017/8.9

\section{References}

Atlas krajiny České republiky: Landscape Atlas of the Czech Republic / red. rada: Ladislav Miko, Peter Mackovčin, et al. - Praha: Ministerstvo životního prostředi České republiky, 2009. - 331 p.

Jenness, J. 2012. Repeating shapes for ArcGIS. Jenness Enterprises.

URL: http://www.jennessent.com/arcgis/repeat_shapes. htm.

Katona. L.F., Wise, T.W. and Holland, C.T.P.: 2018. The South Australian Atlas of Geoscience and Mineral Exploration Data - Woomera Prohibited Area within the 
Gawler Craton, Second Edition, 2018. Department for Energy and Mining. Report Book 2018/00018.

Krylov S.A., Zagrebin G.I. and Loginov, D.S. Atlas mapping: The automated definition of atlases structure // Proceedings of the 7th International Conference on Cartography and GIS, 18-23 June 2018, Sozopol, Bulgaria. Eds: Bandrova T., Konecny M. - Pp. 277-285.

Loginov, D.S. Specific features of using GIS atlases in the geophysical mapping / D.S. Loginov // Proceedings of the 6th International Conference on Cartography and GIS, 13-17 June 2016, Albena, Bulgaria. Eds: Bandrova T., Konecny M. - Pp. 615-623.

Map Portal

(SGIDŠ)

URL: http://mapserver.geology.sk/atlasge/

National Atlas of Spain, 2nd edition (IGN). URL: http://www.ign.es/ane/ane1986-2008/

The National Atlas of Japan (Revised Edition) Geographical Survey Institute, 1990. - 218 p. 